\title{
Factores predictores de la adaptación a la enfermedad en pacientes recién diagnosticadas de cáncer de mama que acuden a un servicio de psicooncología
}

\author{
Sonia Fuentes ${ }^{1}$ y Tomás Blasco ${ }^{2 *}$
}

${ }^{1}$ Unidad de Psico-Oncologia. Institut Català d'Oncología. Hospital Universitari Germans Trias i Pujol. Badalona

${ }^{2}$ Grup d'Investigació en Estrés i Salut GIES. Unitat de Psicologia Bàsica. Universitat Autònoma de Barcelona

\begin{abstract}
Resumen: El trabajo pretende determinar si las preocupaciones de las mujeres recién diagnosticadas de cáncer de mama que reciben atención psicooncológica predicen la adaptación a la enfermedad durante los meses siguientes. Se evaluaron las preocupaciones de 53 mujeres recién diagnosticadas de cáncer de mama antes de comenzar el tratamiento oncológico. La adaptación a la enfermedad se evaluó retrospectivamente, mediante la valoración que realizaron dos psicooncólogas de las historias clínicas de las pacientes. Ninguna preocupación concreta predecía el grado de adaptación de las pacientes, pero el sumatorio total de preocupaciones sí era predictor del nivel de adaptación durante la fase de diagnóstico. El nivel de adaptación durante el tratamiento se relacionaba con estar tomando psicofármacos antes del diagnóstico. El nivel total de preocupaciones y tomar psicofármacos antes del diagnóstico son factores que pueden servir para identificar pacientes que, aún recibiendo atención psicológica, tienen riesgo de mala adaptación durante el diagnóstico y tratamiento oncológico de un cáncer de mama.
\end{abstract}

Palabras clave: Preocupaciones; cáncer de mama; adaptación a la enfermedad; psicofármacos.
Title: Predicting factors of adaptation to illness in recently diagnosed breast cancer patients receiving psychological assistance

Abstract: The aim of this work is to assess whether worries expressed by women with breast cancer recently diagnosed and receiving psychological assistance predict future levels of adaptation to illness. Worries of 53 breast cancer patients were assessed at diagnosis and before starting the oncological treatments. Adaptation to illness was assessed retrospectively by two experienced psychologists reading the patients' clinical reports once these patients finished their treaments. Scores on each worry were not related with adaptation to illness. However, adaptation at diagnosis was predicted by the worries global score, whereas adaptation to illness during the treatment was related with the intake of psychotropic drugs prior to cancer diagnosis. The intensity of worries at diagnosis, and the intake of psychotropic drugs prior to cancer diagnosis, could allow to identify breast cancer patients who, although receiving psychological assistance, are at risk of developing low levels of adaptation to illness during at diagnosis and during the subsequent oncological treatments.

Key words: Worries; breast cancer; adaptation to illness; psychotropic drugs.

proporciones de pacientes que durante el diagnóstico o el tratamiento del cáncer de mama presentan realmente una alteración emocional que precisa de atención profesional oscilan alrededor del 30\% si el malestar emocional se manifiesta a través de un alto grado de ansiedad, y entre el 15\% y el 20\% si se habla de síntomas depresivos (Bárez, 2002; Millar, Purushotham, McLatchie, George, y Murray, 2005; Skarstein, Aass, Fossa, Skovlund, y Dahl, 2000; Söllner, Maislinger, König, DeVries, y Lukas, 2004).

Desde el punto de vista asistencial, sería deseable poder identificar a las pacientes que, en el momento de ingresar en una unidad de oncología para recibir el diagnóstico y/o el tratamiento, presentan un malestar emocional que hace recomendable la intervención psicooncológica, o tienen riesgo de manifestar dicha alteración en un futuro próximo, ya que esta identificación permitiría incrementar la prevención y optimizar la asistencia, al dirigir los recursos de apoyo directamente a las pacientes adecuadas. La literatura ha proporcionado evidencias de diversos factores que guardan relación con la adaptación a la enfermedad, como es la fase de la misma (Frost et al. 2000), el tipo de relación médicopaciente (Mager y Andrykowski, 2002), las variables de personalidad (Millar, Purushotham, McLatchie et al. 2005), y el perfil de las estrategias de afrontamiento empleado (Friedman, Kalidas, Elledge, Chang, Romero, Husain et al. 2006; Sosa, Capafons y Carballeira, 1999).

A partir de estos planteamientos, se ha señalado la necesidad de disponer de instrumentos que permitan detectar los diferentes niveles de malestar de las pacientes a fin de determinar su posible derivación hacia un servicio de atención psicológica o psiquiátrica. Existe una amplia variedad de instrumentos con diferentes características que presentan ven- 
tajas e inconvenientes (Love, 2004), y que deben ser seleccionados en base al objetivo que se persigue con su aplicación, ya que para la identificación de perfiles psicopatológicos su pertinencia no está totalmente aceptada (Sánchez, Peiró y Corbellas, 2008). Recientemente, además, se ha criticado su utilidad (Garssen y DeKok, 2008; Mitchell, Kaar, Coggan y Herdman, 2008) si su aplicación no está integrada dentro de un programa de atención terapéutica.

Si bien la literatura señala que instrumentos tradicionales de screening, como el Hospital Anxiety and Depression Scale (HADS), parecen ser útiles para identificar casos clínicos que requerirían atención psicológica o psiquiátrica (Sellick y Edwarson, 2007; Walker et al. 2007), se han propuesto estrategias más sencillas como el "Distress Thermometer" (Gessler et al. 2008; Hegel, Collins, Kearing, Gillock, Moore y Ahles, 2008; Jacobsen et al. 2005; Zwahlen, Hagenbuch, Carley, Recklitis y Buchi, 2008), que utiliza una escala numérica de 0 a 10 en la que el paciente señala el grado de malestar emocional experimentado durante la última semana, y a la que se sugiere que se acompañe una lista de aspectos sobre los que el paciente indica si han sido, o no, los causantes de dicho malestar. Se han propuesto otras variantes como el "Distress Barometer" (Bauwens, Baillon, Distelmans y Theuns, 2009) o el "Emotion Thermometer" (Mitchell, Baker-Glenn, Granger y Symonds, 2010; Mitchell, Baker-Glenn, Park, Granger y Symonds, 2010), así como la Guía del National Comprehensive Cancer Network (NCCN, 2010). Esta variedad de instrumentos está conllevando una discusión sobre cuáles son los criterios para establecer los puntos de corte adecuados con cada uno de ellos para identificar pacientes que requieren atención psicológica. No obstante, dicha discusión se dirige a la determinación de los pacientes que deben ser derivados hacia la atención psicooncológica, pero, que sepamos, no plantea cómo establecer un perfil de los pacientes que, habiendo sido derivados, y habiendo aceptado la asistencia, pues no hay que olvidar que hay pacientes que rechazan esa posibilidad (Winzer, Hoppe, Altenhoff, $\mathrm{Ku}-$ wert, Koch y Schultz, 2009), tienen riesgo de que su malestar emocional pueda incrementarse a lo largo del tratamiento oncológico.

En ese contexto, el presente estudio plantea la posibilidad de que, en el caso del cáncer de mama, ese perfil de riesgo pueda ser detectado de forma indirecta a través de las preocupaciones expresadas por las pacientes. Las preocupaciones pueden estar reflejando una inquietud que guarde relación directa con el malestar emocional actual y éste puede ser predictor del curso emocional durante los meses siguientes de evolución de la enfermedad (Bennett, Compas, Beckjork y Glinder, 2005; Mehnert y Koch, 2007; Thomas, Thomas, Nandamohan, Nair y Pandey, 2009). Por esta razón, consideramos que las preocupaciones en el momento del diagnóstico podrían ser una guía fiable para detectar a las pacientes que, aún recibiendo atención psicooncológica, tienen riesgo de experimentar bajos niveles de adaptación a la enfermedad, y su evaluación podría efectuarse de manera rápida y poco invasiva a través de un procedimiento sencillo.
El objetivo del presente trabajo es, pues, evaluar si las preocupaciones mostradas en el momento del diagnóstico en pacientes de cáncer de mama que reciben atención psicooncológica pueden servir para detectar a las pacientes que vayan a experimentar un menor grado de adaptación a la enfermedad durante la fase de diagnóstico y durante la fase posterior de tratamiento oncológico. Consideramos que la paciente está adaptada a la enfermedad cuando, de acuerdo con Love (2004), muestra una reactividad emocional adecuada; es decir, cuando la frecuencia, intensidad y duración de emociones como miedo, rabia, ansiedad o tristeza, se pueda considerar como normal frente a la situación provocada por el proceso que conlleva el diagnóstico y tratamiento del cáncer de mama.

Tabla 1. Datos demográficos y médicos de las participantes.

\begin{tabular}{|c|c|c|c|}
\hline & & $N$ & $\%$ \\
\hline \multicolumn{4}{|l|}{ Hospital } \\
\hline & Calella & 39 & 73.58 \\
\hline & Blanes & 14 & 26.42 \\
\hline \multicolumn{4}{|c|}{ Estado Civil } \\
\hline & Casadas/pareja & 42 & 79.24 \\
\hline & Solteras/sin pareja & 11 & 20.76 \\
\hline \multicolumn{4}{|l|}{ Hijos } \\
\hline & Sí & 48 & 90.56 \\
\hline & No & 5 & 9.44 \\
\hline \multicolumn{4}{|c|}{ Tipo de cirugía } \\
\hline & Radical & 17 & 32.07 \\
\hline & Conservadora & 36 & 67.93 \\
\hline \multicolumn{4}{|c|}{ Estadío Clínico } \\
\hline & 0 & 6 & 11.32 \\
\hline & I & 27 & 50.95 \\
\hline & II & 15 & 28.30 \\
\hline & III & 4 & 7.54 \\
\hline & IV & 1 & 1.89 \\
\hline \multicolumn{4}{|c|}{ Forma de detección } \\
\hline & Cribaje & 18 & 33.96 \\
\hline & Atención Primaria & 15 & 28.30 \\
\hline & Hallazgo casual & 20 & 37.74 \\
\hline \multicolumn{4}{|c|}{ Tratamiento oncológico } \\
\hline & Quimioterapia y Radioterapia & 20 & 37.74 \\
\hline & Quimioterapia & 13 & 24.53 \\
\hline & Radioterapia & 15 & 28.30 \\
\hline & Hormonoterapia & 3 & 5.66 \\
\hline & Sin tratamiento & 2 & 3.77 \\
\hline \multicolumn{4}{|c|}{ Psicofármacos antes del diagnóstico } \\
\hline & Sí & 11 & 20.75 \\
\hline & No & 42 & 79.25 \\
\hline
\end{tabular}

\section{Método}

\section{Participantes}

La muestra está compuesta por 53 pacientes recién diagnosticadas de cáncer de mama en el Hospital Sant Jaume, de Calella, (Barcelona) o en el Hospital Comarcal La Selva, de Blanes (Girona), con edades comprendidas entre los 29 y los 82 años y una media de edad de 56.34 años $(S D=12.69)$, que se encontraban en espera del tratamiento quirúrgico una vez 
recibido el diagnóstico oncológico y que eran derivadas al servicio de Psicooncología mediante interconsulta de los médicos de las Unidades de Patología Mamaria, entre septiembre de 2004 y noviembre de 2006. En la Tabla 1 se recogen las características demográficas y médicas de estas pacientes.

\section{Material}

Se elaboró un listado de ítems (listado de preocupaciones) para evaluar el grado de preocupación que producían 13 temas relacionados con la enfermedad (cirugía, anestesia, cicatrices, quimioterapia, alopecia, náuseas, debilidad, radioterapia, molestias de la piel, fatiga, traslado al hospital, pareja, e hijos). Para elaborar el listado de preocupaciones se tuvo en cuenta el elaborado previamente por Bárez (2002) y, a partir del mismo, se concretó una serie de elementos que, dadas las características de los hospitales en los que se iba a llevar a cabo el tratamiento oncológico, y puesto que se iba a trabajar con pacientes que iban a recibir atención psicooncológica, se juzgaron más pertinentes por el equipo investigador, a partir de la experiencia clínica previa en dichos centros con ese tipo de pacientes. Para cada uno de los temas se pedía a la paciente que valorase mediante una escala de 0 a 10 (siendo 0 , nada en absoluto, y 10, la máxima posible) el grado de preocupación que le generaba en ese momento, una vez diagnosticada, y antes de recibir la intervención quirúrgica y/o el tratamiento oncológico neoadyuvante. Se añadieron dos ítems abiertos para que la paciente pudiera expresar preocupaciones respecto a otros temas relacionados o no relacionados con la enfermedad.

\section{Procedimiento}

Una vez aprobado el proyecto de estudio por las correspondientes direcciones de los hospitales, se procedió a incluir en el mismo a todas las pacientes recién diagnosticadas, ya que el protocolo establecía que fueran remitidas mediante interconsulta al Servicio de Psicooncología.

En esta primera visita en el servicio de Psicooncología, se realizaba la recogida de los datos necesarios para la elaboración de la historia clínica psicooncológica y se valoraba la necesidad de llevar a cabo un tratamiento y/o un seguimiento psicooncológico. Como parte del protocolo de recogida de datos para la historia clínica se administraba el listado de preocupaciones, explicándosele a la paciente que esa información se utilizaba para hacer la valoración de su caso y, también, que pasaría a formar parte de un banco de datos para usos de investigación, si ella estaba de acuerdo. Ninguna de las pacientes participantes en el estudio se opuso a que esos datos fueran utilizados para tal fin. A partir de ese momento, las pacientes eran visitadas regularmente en el servicio de Psicooncología hasta que eran dadas de alta en el mismo.

En julio de 2007, una vez que todas las pacientes participantes habían sido dadas de alta en el servicio de psicoonco- logía, el contenido de sus historias clínicas psicooncológicas, fue remitido, en formato anónimo, a dos psicooncólogas expertas, desconocedoras del objetivo de la investigación. En las historias clínicas, cada visita era presentada con un número y una indicación que señalaba si la visita en cuestión pertenecía a la fase de diagnóstico, o al período de tratamiento (ejemplo: "Visita 1/Diagnóstico"; "Visita 3/Tratamiento"). El número de visitas que recibía cada paciente variaba en función de las necesidades de asistencia clínica, por lo que, en cada caso, este número era diferente y osciló entre tres y once siendo el promedio de seis visitas. Las psicooncólogas juezas valoraron de forma global el grado de adaptación en cada uno de esos dos períodos a partir de la lectura de la información contenida en la historia clínica, y que era la que la psicooncóloga del Servicio había considerado importante anotar para registrar el curso clínico de la paciente. Asimismo, se les pidió que valorasen el grado de adaptación a la enfermedad en la visita que consideraron como peor momento dentro de todo el período evaluado (tanto de diagnóstico, como de tratamiento). Para cuantificar el grado de adaptación a la enfermedad se pidió a las dos psicooncólogasjuezas que dieran un valor numérico a partir de una escala 010 en la que 0 indicaba "no ajustada emocionalmente" y 10 indicaba "ajustada emocionalmente". Toda esa valoración se realizó a partir de la siguiente definición de adaptación a la enfermedad, que fue proporcionada junto con las historias clínicas: "Hay adaptación a la enfermedad cuando las reacciones emocionales (miedo, rabia, sentimiento de culpa, ansiedad, tristeza) en la fase de enfermedad en que se encuentra la paciente están dentro de la normalidad en cuanto a frecuencia, intensidad o duración, ya que si estos parámetros se manifestasen en forma excesiva, habria que considerar la existencia de un trastorno adaptativo o de un desajuste emocional".

Una vez recogidas las tres valoraciones que cada una de las juezas hizo sobre cada una de las 53 participantes del estudio, se calculó el grado de adaptación a la enfermedad promediando la puntuación dada por ambas, siempre y cuando la diferencia entre las puntuaciones no fuera superior a 2, criterio adoptado a priori por los investigadores para considerar que las juezas discrepaban. En aquellos casos en que se dio esta discrepancia; es decir, un valor igual o superior a 3 (en total, 14 valoraciones de las 159 posibles que, además, se produjeron en 14 casos diferentes) se requirió la valoración de una tercera psicooncóloga experta y se aceptó el valor dado por ella como magnitud de la adaptación a la enfermedad de la paciente. En total hubo 145 concordancias entre las juezas $(91.1 \%)$. Los valores del índice Kappa fueron de $0.88(\mathrm{p}=.000)$ para el diagnóstico, $0.86(\mathrm{p}=.000)$ para el período de tratamiento, y $0.68(\mathrm{p}=.000)$ para el peor momento, por lo que puede considerarse que el acuerdo interjueces es aceptable.

\section{Análisis estadístico}

Todos los análisis estadísticos se realizaron con el programa SPSS, versión 17.0 para Windows. Para analizar las posibles diferencias en los niveles de adaptación en función 
del estadío clínico de la enfermedad, el tipo de cirugía, el tratamiento oncológico recibido, la forma de detección de la enfermedad y el estar tomando psicofármacos, se utilizó el análisis de la varianza ANOVA. Para analizar la relación entre la adaptación a la enfermedad y la edad, y entre la adaptación a la enfermedad y cada una de las preocupaciones, se ha utilizado la correlación de Pearson. Finalmente, para estudiar la relación entre la adaptación a la enfermedad y las variables explicativas "sumatorio de las preocupaciones" e "ingesta de psicofármacos" se ha efectuado un análisis de regresión lineal múltiple con el método de pasos sucesivos (Stepwise). La significación de todas las pruebas se ha considerado con un nivel de probabilidad del 5\% o inferior, indicándose la significación exacta que ofrecía el paquete estadístico.

\section{Resultados}

La Tabla 2, muestra la intensidad de cada una de las preocupaciones. Las preocupaciones no relacionadas con la enfermedad sólo son indicadas por el $41 \%$ de las pacientes, pero son las que generan una mayor puntuación, alcanzando prácticamente el valor máximo (9.59). Lo que preocupa respecto a temas no relacionados con la enfermedad es el cuidado de personas a cargo (14 casos, 63.6\%) o la adaptación a una fase de viudedad o de duelo (8 casos, 36.3\%). Algo similar sucede con el ítem "otras preocupaciones relacionadas con la enfermedad", que, aun cuando sólo es citado por el $56 \%$ de las pacientes, muestra también un valor muy elevado (9.37), siendo el pronóstico el principal tema indicado por las pacientes (25 casos, 83.3\%), seguido de la preocupación por la pérdida de autonomía (3 casos, 10\%), el tiempo de espera $(1$ caso, $3.3 \%)$ y el grado de afectación en la vida familiar (1 caso, $3.3 \%)$.

La Tabla 3 recoge los niveles de adaptación a la enfermedad de las pacientes en los tres momentos valorados por las juezas. Si consideramos que valores por debajo de 5 indicarían la existencia de una adaptación escasa, el porcentaje de pacientes que se encuentran en esa situación es del $24.5 \%$ (13 casos) en el diagnóstico, el 20.8\% (11 casos) durante el tratamiento, y hasta un $43.4 \%$ (23 casos) en el peor momento.

Los niveles de adaptación a la enfermedad no guardaron relación con la edad de las pacientes, el estadío clínico de la enfermedad, el tipo de cirugía y tratamiento oncológico asignado, o la forma de detección de la enfermedad. Sin embargo, sí hubo una relación entre el hecho de estar tomando psicofármacos, o no, en el momento de ser evaluadas en la primera visita del servicio de psicooncología (Tabla 4), existiendo diferencias estadísticamente significativas en los valores de adaptación a la enfermedad durante los tratamientos $(F=7.25, p=.010)$ y en el peor momento $(F=5.23, p=$ $.026)$, que indican que las pacientes que reciben psicofármacos tienen un peor adaptación a la enfermedad.
Tabla 2. Media y desviación estándar de la intensidad de las preocupaciones

\begin{tabular}{llll}
\hline Preocupación & $N$ & $M$ & $S D$ \\
\hline Molestias en la piel & 53 & 1.66 & 1.81 \\
Traslado al hospital & 53 & 2.34 & 2.90 \\
Radioterapia & 53 & 2.49 & 2.73 \\
Cicatrices & 53 & 2.96 & 3.27 \\
Fatiga & 53 & 3.23 & 3.16 \\
Anestesia & 53 & 3.26 & 3.41 \\
Cirugía & 53 & 3.87 & 3.32 \\
Debilidad & 53 & 4.49 & 3.16 \\
Pareja & 53 & 5.28 & 4.00 \\
Náuseas & 53 & 5.30 & 3.56 \\
Alopecia & 53 & 6.32 & 3.58 \\
Quimioterapia & 53 & 6.60 & 3.38 \\
Hijos & 53 & 7.23 & 3.32 \\
Otros problemas de la enfermedad & 30 & 9.37 & 1.82 \\
Problemas no relativos a la enfermedad & 22 & 9.59 & 1.22 \\
\hline
\end{tabular}

Tabla 3. Puntuaciones de la adaptación en el momento del diagnóstico, durante los tratamientos oncológicos y en el peor momento.

\begin{tabular}{llll}
\hline Momento & $N$ & $M$ & $S D$ \\
\hline Diagnóstico & 53 & 7.08 & 2.01 \\
Tratamiento & 53 & 7.28 & 2.21 \\
Peor momento & 53 & 5.34 & 2.51 \\
\hline
\end{tabular}

No se han encontrado correlaciones estadísticamente significativas entre el nivel de preocupación expresado en cada uno de los ítems y los tres niveles de adaptación a la enfermedad considerados en el estudio. Sin embargo, se elaboró una nueva variable que era el sumatorio de los quince ítems del listado de preocupaciones. Dicha variable puede considerarse como una escala aceptable (alfa de Cronbach $=$ .73) y mostró correlaciones significativas con la adaptación en los tres momentos en que se evaluó: en el diagnóstico $(r=-0.37, \mathrm{p}<.01)$, durante el tratamiento $(r=-0.30 ; \mathrm{p}<.05)$ y en el peor momento $(r=-0.33 ; \mathrm{p}<.05)$. A partir de ahí, y para valorar si esta variable construida como sumatorio de las preocupaciones podía ser el instrumento de detección de niveles bajos de adaptación a la enfermedad, se elaboraron tres modelos de regresión múltiple; uno para cada uno de los niveles de adaptación (en el diagnóstico, durante el tratamiento, y en el peor momento) como variable respuesta, $y$, en todos lo casos, el nivel total de preocupaciones y la ingesta de psicofármacos como variables criterio. Para la adaptación en el diagnóstico, el nivel total de preocupaciones explica el $13.9 \%$ de la varianza $\left(R^{2}=.139 ; F(1,51)=8.26 ; p=.006\right)$, no entrando la ingesta de psicofármacos en el modelo. Para la adaptación durante el tratamiento, la ingesta de psicofármacos explica el $12.5 \%$ de la varianza $\left(R^{2}=.121 ; F(1,51)=7.25\right.$; $p=.010)$, no entrando el nivel total de preocupaciones en el modelo. Finalmente, para la adaptación en el peor momento, vuelve a ser el nivel total de preocupaciones la única variable que entra en el modelo, $(\mathrm{R} 2=.114 ; F(1,51)=6.54 ; p=.014)$, quedando excluida la ingesta de psicofármacos. 
Tabla 4. Puntuaciones de la adaptación en el momento del diagnóstico, durante los tratamientos oncológicos y en el peor momento de las pacientes que toman psicofármacos y de las que no lo hacen.

\begin{tabular}{llllllll}
\hline & \multicolumn{3}{c}{ Diagnóstico } & \multicolumn{2}{c}{ Tratamiento } & \multicolumn{2}{c}{ Peor momento } \\
Psicofármacos & $N$ & $M$ & $S D$ & $M$ & $S D$ & $M S D$ & \\
\hline $\mathrm{Si}$ & 11 & 6.45 & 2.46 & 5.77 & 3.19 & 3.83 & 2.73 \\
$\mathrm{No}$ & 42 & 7.25 & 1.88 & 7.67 & 1.71 & 5.73 & 2.33 \\
\hline
\end{tabular}

\section{Discusión}

Los resultados indican que no hay una preocupación concreta, ni siquiera las que muestran puntuaciones más elevadas, que guarde relación con los niveles de adaptación a la enfermedad. Ni la alopecia, que ha sido señalada como un estresor importante (Lemieux, Maunsell y Provencher, 2008), ni la preocupación por el pronóstico, que es una de las que alcanzan un mayor nivel en la línea de lo encontrado en investigaciones anteriores (Spencer et al. 1999), y de la que podría esperarse, por la temática que contempla, que guardase relación con la adaptación, lo hacen. Es posible que esto pueda deberse a que la mayoría de las pacientes del estudio están en estadíos tempranos, y, por tanto, con buen pronóstico, y que este hecho haya sido asimilado por la paciente en los meses posteriores al diagnóstico, generándole así una mayor tranquilidad respecto a su futuro. En cambio, la intensidad total de las preocupaciones sí muestra relación con la adaptación en el diagnóstico y en el peor momento. Esto pone de manifiesto que no podemos identificar un perfil concreto de preocupaciones entre estas pacientes, y que el nivel de las mismas obedece a razones que cambian en cada caso. La literatura ofrece pocas investigaciones con las que contrastar este hallazgo. Wong-Kim y Bloom (2005) no pudieron identificar ningún factor que predispusiera a manifestar un perfil depresivo, mientras que Wade, Nehmy y Koczwara (2005) señalan que sólo las preocupaciones por la salud en el momento de la cirugía guardan una cierta relación con el malestar emocional posterior. Millar, Purushotham, McLatchie et al. (2005), por su parte, señalaron que los niveles de malestar emocional inmediatamente posterior a la cirugía eran el factor que parecía predecir el distrés en los meses posteriores. Los resultados de la presente investigación estarían de acuerdo con estos dos últimos trabajos, y también con el de Shim, Shin, Jeon y Hahm (2008), ya que cabe considerar que el estado de inquietud provocado por la enfermedad determinaría tanto las preocupaciones como el malestar emocional. Así, el que el nivel total de preocupaciones en el diagnóstico pueda ser predictor del malestar emocional posterior, podría constituir una aportación de interés al ofrecer un elemento de referencia de fácil valoración.

El perfil de riesgo se completa con el hecho de estar tomando psicofármacos, ya que las pacientes que se encuentran en esta situación tienen una peor adaptación durante la fase de tratamiento. Este hecho puede estar indicando que el nivel de malestar previo al diagnóstico es predictor del malestar posterior, tal y como acabamos de señalar al analizar el papel de las preocupaciones. Aun cuando pueda considerar- se que las preocupaciones, en ciertas pacientes, puedan estar mediatizadas por el hecho de tener un malestar emocional que requiere psicofármacos, el que el análisis de regresión muestre que la relación de las preocupaciones con el malestar emocional es independiente del hecho de tomar medicación, refuerza la idea de que aquéllas actúan efectivamente como predictor.

No obstante, el escaso porcentaje de varianza explicado por los modelos de regresión indica que existen otros factores que pueden estar explicando los niveles de adaptación observados. Las creencias sobre la enfermedad, así como la representación que de la misma tienen las pacientes parece guardar relación con el malestar y la calidad de vida (Rozema, Völlink y Lechner, 2009), por lo que deberían ser consideradas en futuras investigaciones con pacientes sometidas a tratamiento psicooncológico.

A partir de estos hallazgos, cabe plantear que la detección de las pacientes de cáncer de mama susceptibles de tener una peor adaptación a la enfermedad debe tener en cuenta el nivel total de preocupación (a partir de la lista elaborada en la presente investigación) y si la paciente está o no tomando psicofármacos en el momento en que recibe el diagnóstico. No obstante, debe tenerse en cuenta que las pacientes evaluadas en esta investigación estaban, en su mayoría, en estadíos tempranos y, además, recibían seguimiento y tratamiento psicooncológico, por lo que no podemos afirmar que las dos variables de cribado identificadas lo sean también en pacientes que no reciban esta intervención. Es ésta una limitación importante del presente trabajo que obliga a ser cautelosos con la posibilidad de generalizar los resultados obtenidos al conjunto de pacientes recién diagnosticadas de cáncer de mama. Además, deben tenerse en cuenta otros aspectos.

En primer lugar, la lista de preocupaciones tiene dos elementos que deberían mejorarse de cara a estudios posteriores que permitieran replicar el que presentamos. Por un lado, la lista de preocupaciones se centra en los síntomas de los tratamientos oncológicos y otros temas de tipo psicosocial, pero no contempla específicamente el pronóstico, que ya hemos indicado que es la principal inquietud respecto a la enfermedad. Por otro lado, los ítems "Debilidad" y "Fatiga" son considerados como sinónimos por muchos pacientes, por lo que podría eliminarse uno de los dos, ya que la correlación entre ambos fue de 0.69 (Fuentes y Blasco, 2010). Finalmente, es asimismo posible que algunos aspectos de tipo laboral, económico, o emocional, que puedan también preocupar a las pacientes, no hayan quedado registrados al no plantearse ítems cerrados específicos para ese tipo de situaciones, aun cuando esta circunstancia estaba contemplada en los ítems abiertos.

En segundo lugar, el contenido de las historias clínicas es muy reducido y no se hacen constar determinadas informaciones confidenciales relatadas durante las visitas de psicooncología, dado que se considera que el acceso a las mismas por parte de otros miembros del equipo de oncología u otros profesionales del hospital que puedan asistir a la pa- 
ciente no es pertinente (las anotaciones sobre los aspectos psicológicos están dentro de la historia clínica de la paciente en todo el ámbito del hospital). Por lo tanto, podría ocurrir que en algunos casos la información fuera escasa y dificultase una evaluación precisa por parte de las juezas. De hecho, estudios como el de Grulke et al. (2005), que utilizan el sistema de evaluación por expertos, usan registros que recogen la totalidad de la información que hay que tener en cuenta para hacer el análisis.

Finalmente, aun cuando hay un grado de acuerdo elevado entre las juezas, los criterios empleados para valorar la existencia de dicho acuerdo han sido consensuados por los autores de la presente investigación, por lo que, de usarse criterios más estrictos, los desacuerdos podrían haber sido mayores. No tenemos elementos para considerar que la discrepancia tolerada en hasta dos puntos de la escala pueda ser totalmente adecuado, pero tampoco podemos afirmar lo contrario. Independientemente de ello, no puede dejarse de lado el hecho de que hay casi un $10 \%$ de desacuerdos, aunque esto podría explicarse por la falta de información de las historias clínicas que describíamos en el párrafo anterior. Por otro lado, si tenemos en cuenta que la prevalencia de pacientes con alteración emocional observada en la literatura está alrededor del 25\% (Bárez, 2002; Millar, Purushotham, McLatchie et al. 2005), y que nuestros resultados también se sitúan en esos valores, podríamos considerar que las valoraciones realizadas por las juezas son adecuadas. No obstante, no hay que olvidar que las pacientes de nuestro estudio no eran, a diferencia de las investigaciones citadas, pacientes

\section{Referencias}

Antoni, M. H., Lechner, S., Kazi, A., Wimberly, S. R., Sifre, T., Urcuyo, K. R., Phillips, K., Gluck, S. y Carver, C. S. (2006). How stress management improves quality of life after treatment for breast cancer. Journal of Consulting and Clinical Psychology, 74(6), 1143-1152.

Bárez, M. (2002). Relación entre percepción de control y adaptación a la enfermedad en pacientes con cáncer de mama. Tesis Doctoral no publicada. Universitat Autònoma de Barcelona. Bellaterra: Barcelona.

Bárez, M., Blasco, T., Fernández-Castro, J. y Viladrich, C. (2007). A structural model of the relationships between perceived control and adaptation to illness in women with breast càncer. Journal of Psychosocial Oncology, 25 (1), 21-43.

Bárez, M., Blasco, T., Fernández Castro, J. y Viladrich, C. (2009). Perceived control and psychological distress in women with breast cancer: a longitudinal study. Journal of Behavioral Medicine, 32, 187-196.

Bauwens, S., Baillon, C., Distelmans, W. y Theuns, P. (2009). The Distress Barometer: validation of method of combining the Distress Thermometer with a rated complaint scale. Psychooncology, 18, 534-542.

Bennett, K., Compas, B. E., Beckjord, E. y Glinder J. G. (2005). Self-blame and distress among women with newly diagnosed breast cancer. Journal of Behavioral Medicine, 28 (4), 313-323.

Cameron, L. D., Booth, R. J., Schlatter, M., Ziginskas, D. y Harman, J. E. (2007). Changes in emotion regulation and psychological adjustment following use of a group psychosocial support program for women recently diagnosed with breast cancer. Psycho-Oncology, 16, 171-180.

Friedman, L., Kalidas, M., Elledge, R., Chang, J., Romero, C., Husain, I., Dulay, M. F. y Liscum, K. (2006). Optimism, social support and psychosocial functioning among women with breast cancer. Psycho-Oncology, 15, 595-603.

Frost, M., Suman, V., Rummans, T., Dose, A. M., Taylor, M., Novotny, P., Johnson, R. y Evans, R. (2000). Physical, psychological and social well- que sólo estaban sometidas al proceso oncológico, sino que estaban recibiendo también atención psicooncológica, por lo que cabe plantearse si realmente dicha atención ha sido suficientemente eficaz, si la definición de adaptación utilizada es imprecisa, o si la resolución del problema del bajo nivel de adaptación requiere de más tiempo para producir resultados, tal como sugiere la investigación de Cameron, Booth, Schlatter, Ziginskas y Harman (2007). Es necesario, por lo tanto, realizar más investigación sobre estos aspectos.

$\mathrm{Al}$ margen de estas limitaciones, a las que hay que añadir que el tamaño de muestra evaluado no es muy grande, y que recoge mayoritariamente a pacientes en estadíos tempranos, creemos que los datos aportados por el presente trabajo ofrecen un marco adecuado para desarrollar futuras investigaciones en este ámbito que permitan clarificar si efectivamente la intensidad de las preocupaciones (cualesquiera que sean) permite detectar a pacientes con cáncer de mama que puedan evolucionar desadaptativamente durante los períodos de diagnóstico y tratamiento. Por otra parte, esta evaluación puede permitir elaborar una intervención terapéutica mucho más personalizada y dirigida a las necesidades de cada paciente, por lo que sugerimos que su valoración se incluya dentro de los protocolos de seguimiento psicooncológico.

Agradecimientos.- Los autores desean manifestar su agradecimiento a Milagros Bárez, Valerie Buscemi, Sandra GarcíaLumbreras y Núria Gondón, por la colaboración prestada en la realización de este trabajo. being of women with breast cancer: the influence of disease phase. Psycho-Oncology, 9, 221-231.

Fuentes, S. y Blasco, T. (2010). Preocupaciones de las pacientes con cáncer de mama que reciben atención psicooncológica. Psicooncología, 7(1), 5160 .

Garssen, B. y De Kok, E. (2008). How useful is a screening instrument? (Letter to the Editor) Psychooncology, 17, 726-728.

Gessler, S., Low, J., Daniells, E., Williams, R., Brough, V., Tookman, A. y Jones, L. (2008). Screening for distress in cancer patients: is the Distress Thermometer a valid measure in the UK and does it measure change over time? A prospective validation study. Psychooncology, 17, 538-547.

González-Tablas, M., Palenzuela, D., Pulido, R., Sáez Regidor, L. y López Pérez, E. (2001). El papel de las expectativas generalizadas de control en el afrontamiento y ajuste psicológico en mujeres con cáncer de mama. Ansiedad y Estrés, 7 (1), 1-14.

Grulke, N., Bailer, H., Hertestein, B., Kächele, H., Arnold, R., Tschuschke, V. y Heimpel, H. (2005). Coping and survival in patients with leukemia undergoing allogeneic bone marrow transplantation, long-therm followup of a prospective study. Journal of Psychosomatic Research; 59, 337-346.

Hack, T. y Degner, L. (2004). Coping responses following breast cancer diagnosis predict psychological adjustment three years later. PsychoOncology, 13, 235-247.

Hegel, M. T., Collins, E. D., Kearing, S., Gillock, K. L., Moore, C. P. y Ahles, T. A. (2008). Sensitivity and specificity of the Distress Thermometer for depression in newly diagnosed breast cancer patients. Psychooncology, 17, 556-560.

Jacobsen, P. B., Donovan, K. A., Trask, P. C., Fleishman, S. B., Zabora, J., Baker, F. y Holland, J. (2005). Screening for psychologic distress in ambulatory cancer patients: a multicenter evaluation of the distress thermometer. Cancer, 103, 1494-1502. 
Lemieux, J., Maunsell, E. y Provencher, L. (2008). Chemotherapy-induced alopecia and effects on quality of life among women with breast cancer: a literature review. Psycho-Oncology, 17, 317-328.

Love, A. (2004). The identification of psychological distress in women with breast cancer. Melbourne: National Breast Cancer Centre.

Mager, W. y Andrykowski, M. (2002). Communication in the cancer "Bad News" consultation: patient perceptions and psychological adjustment. Psycho-Oncology, 11, 35-46.

Mehnert, A. y Koch, V. (2007). Prevalence of acute and post-traumatic stress disorder and comorbid mental disorders in breast cancer patients during primary cancer care:A prospective study. Psycho-Oncology, 16, 181 188.

Millar, K., Purushotham, A.D., McLatchie, E., George, W. D. y Murray, G. D. (2005). a 1-year prospective study of individual variation in distress and illness perceptions after treatment for breast cancer. Journal of Psychosomatic Research, 58, 335-342.

Mitchell, A. J., Baker-Glenn, E. A., Granger, L. y Symonds, P. (2010). Can the Distress Thermometer be improved by additional mood domains? Part I. Initial validation of the Emotion Thermometers tool. Psychooncology, 19, 125-133.

Mitchell, A. J., Baker-Glenn, E. A., Park, B., Granger, L. y Symonds, P. (2010). Can the Distress Thermometer be improved by additional mood domains? Part II. What is the optimal combination of emotion thermometers?. Psychooncology, 19, 134-140.

Mitchell, A. J., Kaar, S., Coggan, C. y Herdman, J. (2008). Acceptability of common screening methods used to detect distress and related mood disorders - preferences of cancer specialists and non-specialists. Psychooncology, 17, 226-236.

National Comprehensive Cancer Network (2010). Clinical Practice Guidelines in Oncology Distress management V.1.2010. Recuperado el 1 de junio de 2010 de

http://www.nccn.org/professionals/physician_gls/PDF/distress.pdf

Rozema, H., Völlink, T. y Lechner, L. (2009). The role of illness representations in coping and health of patients treated for breast cancer. Psychooncology, 18, 849-857.

Sánchez, P. T., Peiró, G. y Corbellas, C. (2008). ¿Evaluación de la psicopatología a través de escalas? Psicooncología, 5(1), 71-81.

Sellick, S. M. y Edwardson, A. D. (2007). Screening new cancer patients for psychological distress using the Hospital Anxiety and Depression Scale. Pyschooncology, 16, 534-542.
Shim, E., Shin, Y., Jeon, H. y Hahm, B. (2008). Distress and its correlates in Korean cancer patients: pilot use of the distress thermometer and the problem list. Psycho-Oncology, 17(6), 548-555.

Skarstein, J., Aass, N., Fossa, S. D., Skovlund, E. y Dahl, A.A. (2000). Anxiety and depression in cancer patients: relation between the Hospital and Anxiety Depression Scale and the European Organization for Research and Treatment of Cancer Core Quality of Life Questionnaire. Journal of Psychosomatic Research, 49, 27-34.

Sirgo, A., Díaz-Ovejero, M. B., Cano-Vindel, A. y Pérez-Manga, G. (2001). Ansiedad, ira y depresión en mujeres con cáncer de mama. Ansiedad Estrés, 7 (2-3), 259-271.

Söllner, W., Maislinger, S., König, A., DeVries, A. y Lukas, P. (2004). Providing pyschosocial support for breast cancer patients based on screening for distress within a consultation-liaison service. Psycho-Oncology, 13, 893-897.

Sosa, C. D., Capafons, J. I. y Carballeira, M. (1999). Afrontamiento, ajuste psicológico y cáncer de mama. Ansiedad y Estrés, 5 (1), 47-60.

Spencer, S. M., Lehman, J. M., Wynings, C., Arena, P., Carver, C. S., Antoni, M. H., Derhagopian, R. P., Ironson, G. y Love, N. (1999). Concerns about breast cancer and relations to psychosocial well-being in a multiethnic sample of early stage patients. Health Psychology, 18, 159-168.

Thomas, B. C., Thomas, I., Nandamohan, V., Nair, M. K. y Pandey, M.(2009). Screening for distress can predict loss of follow-up and treatment in cancer patients: results of development and validation of the Distress Inventory for Cancer Version 2. Psychooncology, 18, 524-533.

Wade, T. D., Nehmy, T. y Koczwara, B. (2005). Predicting worries about health after breast cancer surgery. Psycho-Oncology, 14, 503-509.

Walker, J., Postma, K., McHugh, G. S., Rush, R., Coyle, B., Strong, V. y Sharpe, M. (2007). Performance of the Hospital Anxiety and Depression Scale as a screening tool for major depressive disorder in cancer patients. Journal of Psychosomatic Research, 63, 83-91.

Winzer, A., Hoppe, A., Altenhoff, J., Kuwert, C., Koch, U. y Schultz, H. (2009). Interest in a psycho-educational group intervention among outpatients with malignant melanoma in relation to their need: which patients are likely to participate? Psychooncology, 18, 1179-1188.

Wong-Kim, E. y Bloom, J. (2005). Depression experienced by young women newly diagnosed with breast cancer. Psycho-Oncology, 14, 564-573.

Zwahlen, D., Hagenbuch, N., Carley, M. I., Recklitis, C. J. y Buchi, S. (2008). Screening cancer patients' families with the Distress Thermometer (DT): A validation study. Psychooncology, 17, 959-966.

(Artículo recibido: 26-11-2010, revisado: 14-12-2011, aceptado: 31-12-2011) 